\section{Combined ectopic expression of Pdx1 and Ptf1a/p48 results in the stable conversion of posterior endoderm into endocrine and exocrine pancreatic tissue}

\author{
Solomon Afelik, Yonglong Chen, \\ and Tomas Pieler ${ }^{1}$
}

Georg-August-Universität Göttingen, Zentrum Biochemie und Molekular Zellbiologie, Abteilung Entwicklungsbiochemie, 37077 Göttingen, Germany

Patterning of the embryonic endoderm into distinct sets of precursor cells involves the precisely regulated activities of key transcription regulators. Ectopic, pan-endodermal activation of $\mathrm{XPtf1a/p48}$ during pancreas precursor cell stages of Xenopus embryogenesis results in an expansion of the pancreatic territory, precisely within the borders of XlHbox 8 expression. A combination of both activities is sufficient to expand the pancreatic precursor cell population also into more posterior portions of the endoderm. Both treatments result in the formation of a giant pancreas that persists up to late tadpole stages of development and carries both supernumerary endocrine and exocrine cells. A combination of XPtf1a/ p48 and XlHbox8 is thus sufficient to convert nonpancreatic endodermal cells into pancreatic precursor cells.

Supplemental material is available at http://www.genesdev.org.

Received January 4, 2006; revised version accepted March 9, 2006.

The vertebrate pancreas develops from one dorsal and two ventral evaginations in the endodermal epithelium (Slack 1995). Early pancreatic precursor cells express Pdx1, a homeodomain protein that is also expressed in the adjacent presumptive stomach and duodenum (Ohlsson et al. 1993; Jonsson et al. 1994), as well as Ptf1a/p48, a basic helix-loop-helix (bHLH) transcription factor (Kawaguchi et al. 2002). Lineage tracing studies have indicated that the $P d x 1$-expressing cells represent precursor cells that contribute to the formation of both endocrine and exocrine lineages of the mature pancreas (Gu et al. 2002). In Pdx1-homozygous mutant mice, pancreatic buds seem to be arrested in their development; however, early glucagon- and insulin-expressing cells can still be detected (Ahlgren et al. 1996; Offield et al. 1996). Such mice exhibit additional malformations in stomach and duodenum, where $P d x 1$ is normally expressed. Interestingly, and of primary relevance for the

[Keywords: Xenopus laevis; pancreas development; Ptf1a/p48; transspecification]

${ }^{1}$ Corresponding author.

E-MAIL tpieler@gwdg.de; FAX 0049-551-3914614.

Article is online at http://www.genesdev.org/cgi/doi/10.1101/gad.378706. results reported in this communication, ectopic expression of $P d x 1$ in nonpancreatic chicken endoderm resulted in the initiation of pancreatic budding, but it was not sufficient to promote differentiation of either exocrine or endocrine cells (Grapin-Botton et al. 2001). Ptfla/p48 is a bHLH transcription factor, which was originally identified as a part of a heterotrimeric pancreas transcription factor complex, referred to as PTF1 that activates transcription of exocrine specific pancreatic genes in the mature pancreas (Cockell et al. 1989. Beres et al. 2006). Mice bearing a null mutation of Ptf1a/ p48 are completely devoid of exocrine pancreas, while endocrine pancreatic cells still form, but are found to be translocated to the spleen (Krapp et al. 1998). More recent studies have revealed that Ptf1a/p48 is already expressed in pancreatic precursor cells which contribute to all pancreatic cell types, and that, in the absence of Ptfla/p48, pancreatic precursor cells adopt a duodenal fate (Kawaguchi et al. 2002). These findings suggest a role for Ptf1a/p48 that is not solely in exocrine differentiation, but that is also relevant for cells of the endocrine lineages.

Here, we report on observations that are in strong support of the concept that a combination of these two transcription factors, XlHbox 8 and XPtf1a/p48, is sufficient for the commitment of endodermal cells to a pancreatic fate in Xenopus embryos.

\section{Results and Discussion}

Within the endoderm, expression of XPtf1a/p48 is specifically restricted to the entire dorsal and ventral pancreatic anlagen as early as at stage 27 , while expression of X1Hbox8 also includes adjacent regions of the presumptive stomach and duodenum (Fig. 1). To test for the function of XPtfla/p48 in the context of early pancreas development, mRNA encoding a dexamethasone-inducible variant of XPtf1a/p48, referred to as XPtf1a/p48GR, was injected into all four vegetal blastomeres of eightcell-stage Xenopus embryos. Following the temporal expression profile of the endogenous gene, dexamethasone treatment was performed at embryonic stage 27. Coinjection of $\beta$-galactosidase-encoding mRNA indicates that the injected RNA is evenly distributed throughout the entire endoderm at tadpole stages (Supplementary Fig. 1). As a result, we observed ectopic expression of exocrine pancreatic differentiation markers, such as pancreatic protein disulphide isomerase (XPDIp) and elastase (Fig. 2A, panels 3, 6); other exocrine marker genes like amylase, carboxypeptidase, and trypsinogen showed similar effects (data not shown). Embryos that were injected with the XPtf1a/p48GR mRNA, but not treated with dexamethasone, showed no such effect, and they were indistinguishable from uninjected embryos treated with dexamethasone (Fig. 2A, panels 2, 5). Strikingly, the ectopic expression of exocrine pancreatic genes is specifically restricted to the territory of prospective stomach and duodenum, adjacent to the developing pancreas.

Insulin expression is first detected exclusively in the dorsal pancreas by stage 32 , representing the only endocrine differentiation gene expressed before stage 45 (Kelly and Melton 2000; Horb and Slack 2002). Insulin expression remained unaffected in embryos overexpress- 


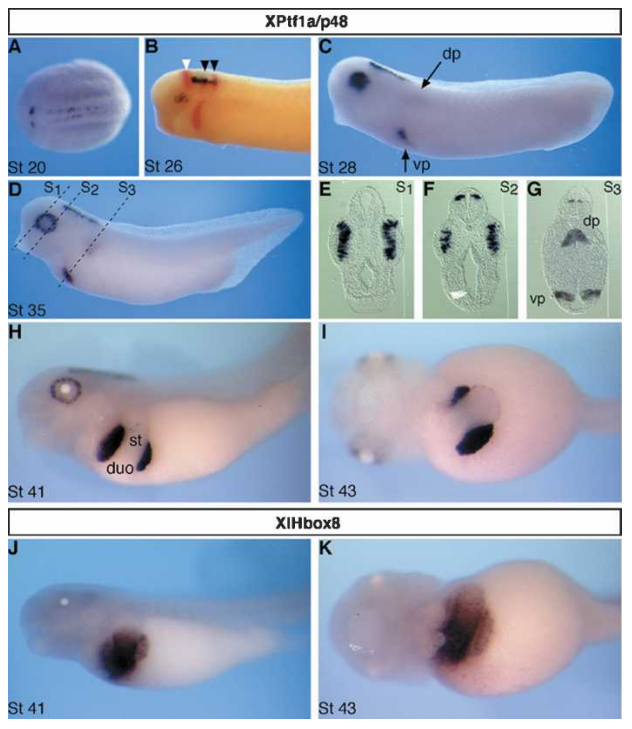

Figure 1. Whole-mount in situ hybridization analysis of XPtf1a/ p48 expression during Xenopus embryogenesis and in comparison to XlHbox8. (A) Dorsal view of a stage 20 embryo, anterior toward the left. XPtf1a/p48 transcripts (blue) are detected along two parallel longitudinal stripes representing the neural folds. $(B)$ Double-staining in situ hybridization of tailbud-stage (stage 26) embryos using En2 (red) as a midbrain-hindbrain boundary marker (white arrowhead) and Krox20 (red) as a marker for rhombomeres 3 and 5 (black arrowheads). Neural expression of XPtf1a/p 48 becomes restricted to the hindbrain, with the anterior end defined by the midbrain-hindbrain boundary and the posterior limit by rhombomere 5. XPtf1a/ p48 transcripts are also becoming detectable in the developing retina. During later phases of development, retinal expression is confined to the proliferating precursor cells of the ciliary marginal zone and expression in the neural tube to dorsal elements (shown in $E, F) .(C, D)$ Lateral view of stage 28 and stage 35 embryos stained for XPtf1a/p48 expression. (E-G) Transverse sections (S1, S2, S3) of a stage 35 embryo at the levels indicated in $D$, dorsal to the top. $(H)$ Lateral view. (I) Ventral view. $(J, K)$ Lateral and ventral view of XlHbox8 expression. (duo) Duodenum; (dp) dorsal pancreatic bud; (st) stomach; (vp) ventral pancreatic buds.

ing XPtf1a/p48GR (Fig. 2A, panel 9). Similarly, examination of embryos at stages 41 and 43 showed no obvious effect on insulin expression (data not shown).

In normal embryos, the Forkhead transcription factor Foxal is expressed in the stomach and duodenum but excluding the pancreas, as well as in the proctodaeum (Fig. 2A, panel 10). Upon ectopic activation of XPtf1a/ p48 in the endoderm, expression of Foxa1 is lost specifically at the site of ectopic pancreatic gene expression, whereas its expression in the proctodaeum remains unaffected (Fig. 2A, panel 12). These findings suggest adaptation of a pancreatic fate with a concomitant loss of duodenal and stomach identities within the X1Hbox8 positive territory, rather than the induced pancreas expansion being a consequence of increased proliferation.

In agreement with previous studies (Grapin-Botton et al. 2001), we have also found that ectopic expression of $P d x 1$ in the endoderm of Xenopus embryos is not sufficient to cause ectopic pancreas differentiation (data not shown). The extent of ectopic gene induction observed for exocrine pancreatic genes in XPtf1a/p48GR-injected embryos was restricted to regions of the foregut endoderm reminiscent of the expression domain for XlHbox8. This situation prompted us to test whether the combined activities of $P t f 1 a / p 48$ and X1Hbox 8 are sufficient to convert nonpancreatic into pancreatic endoderm. Coinjection of XPtf1a/p48GR with XlHbox8GR results in an induction of exocrine-specific differentiation genes also outside of stomach and duodenal regions, now expanding into the more posterior portions of the endoderm (Fig. 2B, panel 3). The occurrence of several discrete foci of ectopic pancreatic gene expression provides a further argument for fate conversion rather than expansion of a primary pancreatic territory by increased proliferation being responsible for the effects observed. The expression of insulin in the dorsal pancreas of early (stage 36) embryos remained again unaffected (Fig. 2B, panel 6). In all cases of ectopic pancreatic gene induction, the onset of expression in the ectopic domain followed the normal temporal pattern, as observed in uninjected control embryos (data not shown).
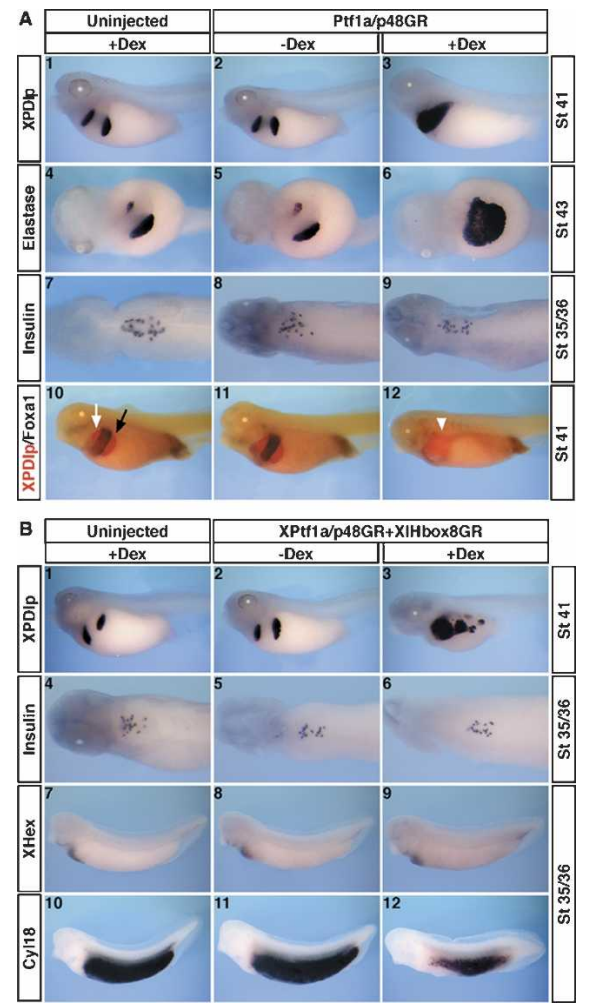

Figure 2. A combination of XPtf1a/p48 and XlHbox8 induces ectopic pancreatic differentiation. (A) Whole-mount in situ hybridization analysis of a panel of markers reveals that ectopic expression of XPtf1a/p48 converts part of the presumptive stomach and duodenum into a pancreatic fate. (Panels 1-3) Lateral view. Ninety-three percent of the embryos examined showed effects as in panel 3 $(n=62)$. (Panels 4-6) Ventral view. Phenotype in panel 6, 87\%, $n=24$. (Panels 7-9) Dorsal view (head toward the left) after removal of somites, neural tube, and notochord. As shown in panel 9, none of the embryos examined exhibited altered insulin expression $(n=25)$. (Panels 10-12) Lateral view. Double-staining in situ hybridization with Foxa1 (blue) and XPDIp (red). (Panel 10) Black and white arrows indicate dorsal and ventral pancreas, respectively. (Panel 12) The white arrowhead indicates ectopic XPDIp and loss of Foxa1 expression $(85 \%, n=67)$. (B) Combined overexpression of XPtf1a/p48 and X1Hbox8 leads to a reduction of intestinal marker gene expression and concomitant ectopic expression of exocrine pancreatic marker genes in the presumptive intestine. (Panels 1-3,7-12) Lateral view. Phenotype in panel $3,82 \%, n=45$; phenotype in panel $12,70 \%, n=53$. (Panels 4-6) Dorsal view (head toward the left) after removal of somites, neural tube, and notochord. 
In order to analyze if regions of ectopic pancreatic gene expression lose their normal molecular identity, we examined the expression of the intestinal differentiation marker Cyl18 (Chen et al. 2003). In control embryos Cyl18 is expressed in the entire intestinal region (Fig. 2B, panel 10), but not in the stomach and duodenum. Ectopic activation of XPtf1a/p48GR together with XlHbox8GR results in a loss of Cyl18 expression in regions of the intestine posterior to the normal position of the pancreas (Fig. 2B, panel 12), while expression of the liver marker XHex was unaffected (Fig. 2B, panel 9), suggesting a restricted regional competence of the endoderm to undergo the fate switch. Thus, results obtained with both injection of XPtf1a/p48 alone or together with X1Hbox8, are compatible with the idea that it is a combination of both activities that defines pancreatic precursor cells in the endoderm of Xenopus embryos that have the ability to differentiate accordingly.

The above embryo injection experiments had employed dexamethasone treatment at embryonic stage 27 to induce ectopic activities when the corresponding endogenous genes start to be expressed in the developing pancreas. In order to test when exactly ectopic activation of XPtf1a/p48GR is required for the expansion of the pancreatic territory, a systematic variation of the timepoint of hormone treatment was performed (Fig. 3A). Ectopic XPtf1a/p48GR operates most efficiently during pancreatic precursor cell stage (stage 27), which is when endogenous X1Hbox8 and XPtf1a/p48 are present, but before pancreatic differentiation markers start to be expressed.

In order to test if the effects observed are transient or stable, embryos injected with either XPtf1a/p48GR alone or in combination with $X 1 H b o x 8 G R$ were grown to late tadpole stages (stage 48) and analyzed for the expression of XPDIp, insulin, and glucagon. Morphology and marker gene expression reveal a dramatic expansion of the pancreas. Conversely, stomach and duodenum are deformed and reduced in size (Fig. 4). The giant pancreas consists mainly of exocrine cells with interspersed endocrine cells, in a ratio similar to the pancreas in control embryos (Fig. 3B).

The requirement for XPtf1a/p48 in the context of pancreas development in Xenopus was tested by microinjection of the corresponding morpholino antisense oligonuleotide (MO). The specificity of MOs employed in this study was tested using both in vitro and in vivo assays (Supplementary Figs. 2, 3). Manipulated embryos displayed a complete loss of exocrine differentiation gene expression, such as XPDIp, amylase, and trypsinogen. Conversely, early insulin expression was maintained under the same experimental conditions in the MO-injected embryos, but the insulin-expressing cells were clustered and not dispersed as in the control embryos (Fig. 5A; data not shown). Interestingly, the secondary wave of endocrine differentiation that is clearly visible in stage 48 control embryos, does not occur in XPtf1a/p48 knockdown embryos; the pancreatic tissue seems to be completely lost since insulin or glucagon expression cannot be detected in serial transversal sections that cover the entire length of the embryo (Fig. 5B; data not shown).

Embryos injected with MO directed against X1Hbox8 revealed similar effects as obtained with the Ptf1a/p48 MO (i.e., loss of exocrine marker gene expression), while the early dorsal expression of insulin was maintained.
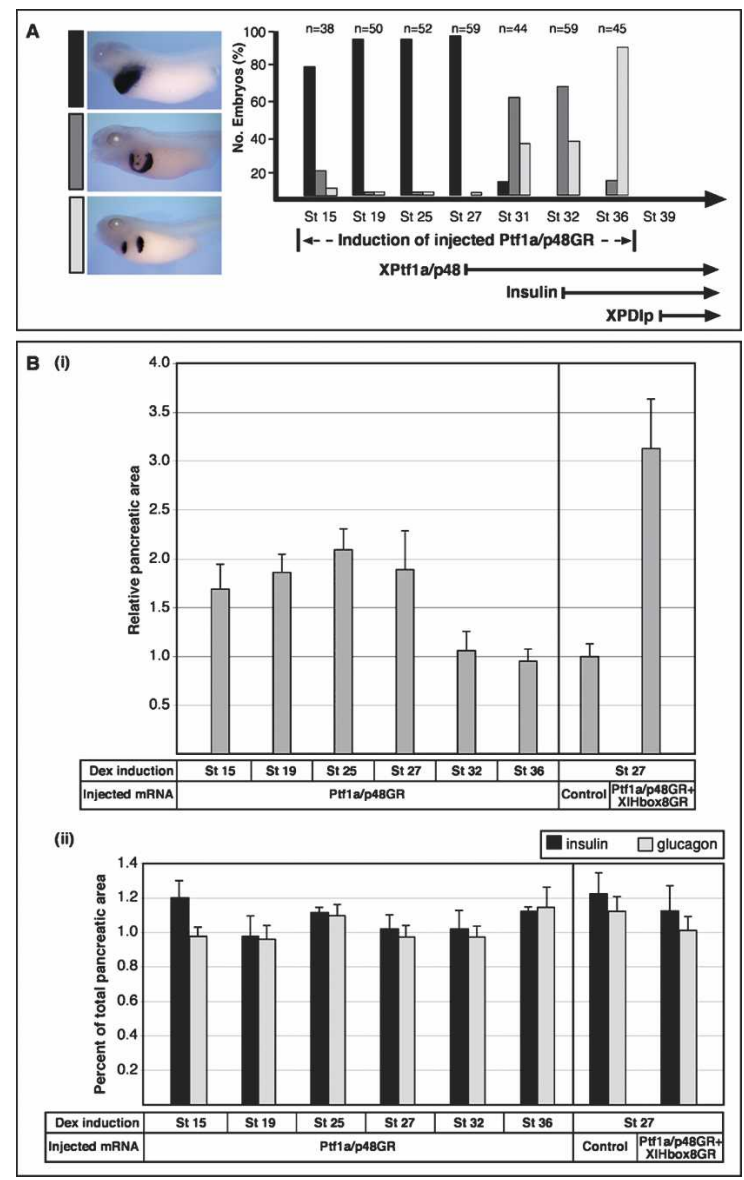

Figure 3. The Ptf1a/p48-mediated increase of ectopic exocrine and late endocrine cells requires uncommitted endoderm. Dexamethasone induction of injected embryos was performed at different time points in between stage 15 and stage 36 , as indicated. The effects on pancreas development were evaluated by whole-mount in situ hybridization analysis of XPDIp expression at stage 41 for the exocrine pancreas, and double-staining immunohistochemical analysis of insulin and glucagon expression at stage 48 for the endocrine pancreas. For morphometric quantification, the pixel number of insulin-positive and glucagon-positive cells was measured separately in serial sections using Adobe. $(A)$ The extent of ectopic XPDIp expression at stage 41 is dependent on the stage of induction of injected Ptf1a/ p48GR. The bottom right part illustrates the temporal expression profile of endogenous XPtf1a/p48, insulin, and XPDIp. (B) Despite an increase in total pancreatic area, there is no significant difference in the ratio of endocrine to exocrine pancreatic cells in embryos overexpressing Ptf1a/p48 alone or in combination with X1Hbox8. (Panel i) Ectopic expression of Ptf1a/p48GR alone or in combination with XlHbox8GR results in a roughly two- and threefold increase in total pancreatic area relative to uninjected control embryos, respectively. (Panel ii) No significant difference is observed for the ratio of endocrine to total pancreatic area in a comparison of different time points for dexamethasone treatment. Sectioned pancreatic tissue was immunostained for insulin and glucagon, nuclei were counterstained with DAPI. Boundaries of the pancreatic area were delineated on the basis of morphology and pixel quantification was performed using Adobe Photoshop. An entire series of sections was analyzed for eight different embryos in each experiment. The average total pixel number of a series of pancreatic sections from control embryos is referred to as 1 .

Even more unexpectedly, microinjection of a combination of both XlHbox 8 and Ptf1a/p48 MOs again results in the same situation (i.e., loss of exocrine and maintenance of early insulin expression) (Supplementary Fig. 4). 
Taken together, these observations indicate that both XPtfla/p48 and XlHbox8 are needed to induce the pancreatic lineage within the endoderm. It appears however, as though the early insulin expression commencing at stage 32 is independent of the same two proteins.

\section{Concluding remarks}

The onset of Ptf1a/p48 expression corresponds in time with the expression of $P d \times 1 / X 1 H b o x 8$, suggesting that it is a combination of both activities that defines the pancreatic precursor cell status in the anterior endoderm of vertebrates (Kawaguchi et al. 2002; Bort and Zaret 2002). Supporting this hypothesis, ectopic expression of $X P t f 1 a / p 48$ alone results in the expansion of pancreatic differentiation into the $X 1 H$ box8-positive territory outside of the pancreatic rudiments that normally develops into stomach and duodenum. Conversely, ectopic expression of XlHbox8 fails to induce ectopic pancreas differentiation in Xenopus embryos. Very much in line with these latter results, previous studies using in ovo electroporation of chick embryos had shown that ectopic expression of $P d x 1$, the homolog of $X 1 H b o x 8$, results in the initiation of epithelial budding, but these structures fail to complete pancreatic cytodifferentiation (GrapinBotton et al. 2001).

It was also reported that adenovirus-mediated $P d x 1$ gene transfer into the adult liver induces both endocrine

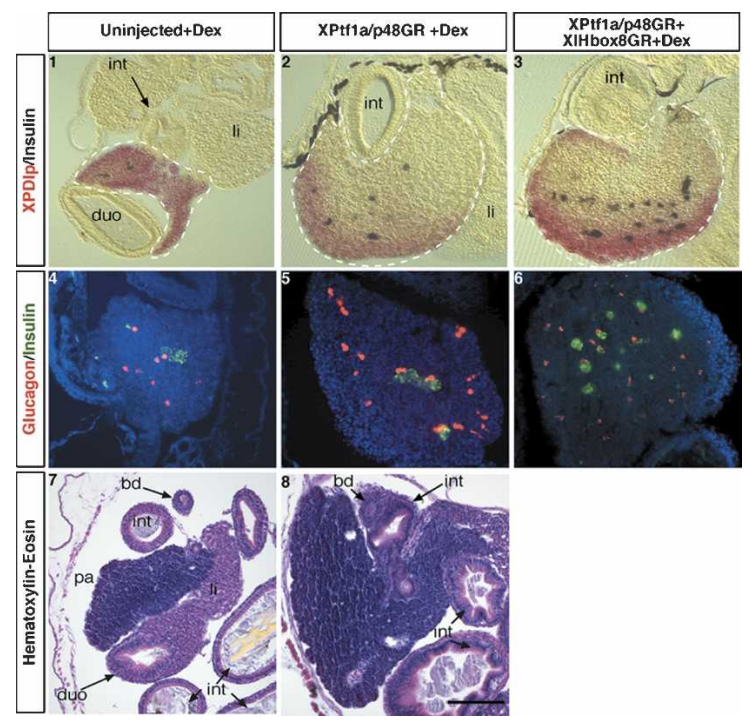

Figure 4. Giant pancreata containing both differentiated endocrine and exocrine cells at late tadpole stage are generated by ectopic expression of XPtf1a/p48GR alone or in combination with XlHbox8GR. (Panels 1-3) Vibratome sections of stage 48 embryos (microinjected as indicated and dexamethasone-treated at stage 27) after double-staining whole-mount in situ hybridization (insulin in blue, XPDIp in red). Due to incomplete probe penetration into the giant pancreas, the inner part remains refractory to the staining procedure; the boundaries of pancreatic tissue (broken line) were identified by microscopic analysis. Phenotype in panel 2, 60\%, $n=15$; in panel 3, 66\%, $n=12$. (Panels 4-6) Double-staining, immunohistochemical analysis for insulin (green) and glucagon (red) expression. In panel 5, 70\%, $n=10$; in panel 6, 62\%, $n=8$ (morphometric quantification, see Fig. 3). Bar, $200 \mu \mathrm{m}$. (Panels 7,8) Histological analysis (hematoxylin and eosin staining) of pancreas and neighboring tissues. In panel $8,58 \%, n=12$. (bd) Bile duct; (duo) duodenum; (int) intestine; (li) liver; (pa) pancreas.

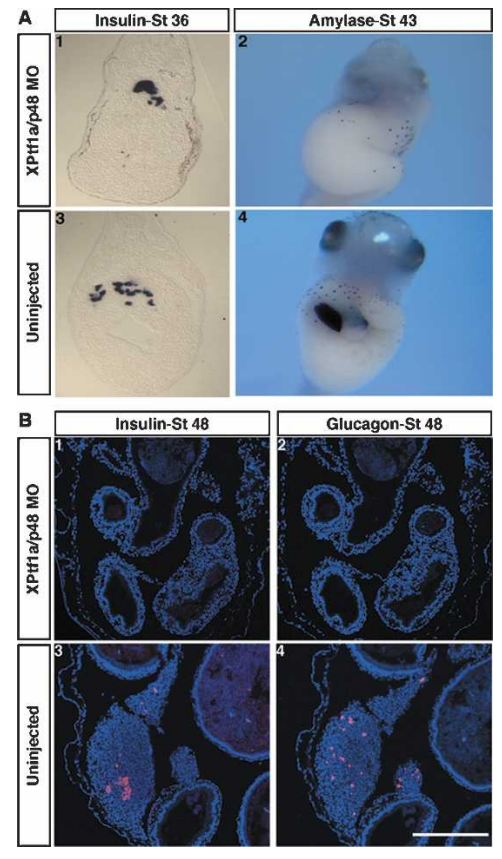

Figure 5. Early insulin expression is independent of XPtf1a/p48, while later pancreatic insulin and glucagon expression depends on $\mathrm{XPtf1a/p48.} \mathrm{(A)} \mathrm{Knockdown} \mathrm{of} \mathrm{XPtf1a/p48} \mathrm{by} \mathrm{antisense} \mathrm{MO} \mathrm{injec-}$ tion results in a loss of exocrine pancreatic gene expression, while early dorsal insulin expression remains unaffected. (Panels 1,3) Transverse vibratome sections (panel 1, 100\%, $n=85$; morphometric quantification of insulin expression, average pixel number in panel 1, 12,265 \pm 5152; panel 3, 15,537 \pm 2108 ). (Panels 2,4) Ventral view, head toward the top (panel $2,77 \%, n=27)$. (B) The second phase of endocrine pancreatic differentiation is blocked upon knockdown of XPtf1a/p48 by antisense MO injection. Immunohistochemical analysis of insulin and glucagon expression in uninjected control (panels 3,4) and in XPtf1a/p48 MO-injected embryos (panels $1,2)$ at stage 48 of development. Phenotype in panels 1 and 2,60\%, $n=15$. Bar, $200 \mu \mathrm{m}$.

and exocrine pancreatic gene expression in the mouse (Ferber et al. 2000; Ber et al. 2003; Kojima et al. 2003), and that an activated version of $P d x 1(P d \times 1-V P 16)$, under the control of the liver-specific transthyretin promoter, is sufficient to transform the liver into ectopic pancreatic tissue in transgenic Xenopus embryos (Horb et al. 2003). Such transdifferentiation events are conceptually different from what we report in this communication; ectopic Pdx1 activity is activated in differentiated cells rather than in multipotent embryonic precursor cells. In our experiments, liver development was not found to be affected by the early embryonic activation of a combination of XPtf1a/p48 and XlHbox8, as evidenced by use of $X H e x$ as a liver-specific marker gene. Additionally, we did not observe pancreatic gene expression in the embryonic liver of such manipulated embryos.

Using antisense MO injection, we demonstrate that inhibition of Ptf1a/p48 translation in Xenopus embryos results in a specific loss of the entire exocrine pancreas, which is in line with Ptf1a/p48 loss of function results in other systems (Krapp et al. 1998; Kawaguchi et al. 2002; Lin et al. 2004; Zecchin et al. 2004). In respect to effects on endocrine differentiation, where early dorsal insulin expression was not affected in frog embryos, while the later differentiation of insulin- and glucagon-producing pancreatic cells was blocked, the situation is more com- 
plex in a comparison of the different systems. In Ptf1a/ p48 knockout mice, only a small number of endocrine cells differentiate apparently normally but are mislocated to the spleen (Krapp et al. 1998; Kawaguchi et al. 2002), while in the zebrafish, the differentiation of the principal islet is unperturbed (Lin et al. 2004; Zecchin et al. 2004), but late appearing, anterior endocrine cells are greatly diminished (Lin et al. 2004). Thus, with the distinction of effects on early versus late endocrine differentiation, these latter results compare most closely to the situation in frog embryos. Early differentiating endocrine cells also persist upon inhibition of $\mathrm{X} 1 \mathrm{Hbox} 8$ activity by antisense $\mathrm{MO}$, a finding that is reminiscent of the survival of early insulin- and glucagon-expressing cells in $P d x 1^{-/-}$mice (Ahlgren et al. 1996). Pdx1 knockdown in the zebrafish perturbs both endocrine and exocrine pancreas development, including a severe reduction in respect to the immunoreactive insulin (Yee et al. 2001). The early dorsal, insulin-expressing cells in Xenopus embryos can be detected as early as stage 32, which is prior to pancreas morphogenesis (Kelly and Melton 2000). It seems, therefore, that the second wave of pancreas differentiation, which involves the vast majority of endo- and exocrine marker genes, has to be treated separately from the events that result in the dorsal, early insulin-expressing cells in Xenopus embryos.

\section{Materials and methods}

Isolation and construction of inducible XPtf1a/p48 and X1Hbox8 proteins

Degenerate primers $\left(5^{\prime}\right.$-TCYATYAACGAYTTCGAG-3' and $5^{\prime}$-GTCCT CKGGKGTCCASCCYT-3') based on the conserved domains of the human, rat, mouse, and zebrafish XPtf1a protein sequences (GenBank accession nos. CAI12668, NP_446416, NP_061279, and AAO92259, respectively) were used to generate a partial Xenopus Ptf1a/p48 (XPtf1a/p48) cDNA fragment by RT-PCR. The 5'- and 3'-extended sequences were isolated from an adult Xenopus pancreas $\lambda$ ZAP Express phage cDNA library (Afelik et al. 2004) by PCR screening. The full-length XPtf1a/p48 (GenBank accession no. DQ007931) ORF was cloned into the pCS2+ vector (Rupp et al. 1994) and fused with the human glucocorticoid receptor (GR) ligand-binding domain (Gammill and Sive 1997), and is referred to as XPtf1a/p48GR. A corresponding murine Ptf1a/p48GR fusion construct in pCS2+ was generated using the mouse Ptf1a/p48 cDNA clone (Obata et al. 2001). The full-length X1Hbox8 ORF was amplified by RT-PCR using the data bank sequence (GenBank accession no. X16849) and similarly cloned into the pCS2+ vector-containing $G R$ ligand-binding domain.

\section{Embryo microinjection}

Albino Xenopus laevis embryos were obtained by hormone-induced egg laying and in vitro fertilization. For knockdown experiments, 2 pmol of MO (GeneTools, LLC) directed against either XPtf1a/p48 (5'-CCAACT GCTCCAGGACCGTTTCCAT-3') or XlHbox8 (5'-TATTGGTCATCA GCATTCATGGCGA-3') were injected per embryo. In the case of combined injections of both MOs, $1 \mathrm{pmol}$ of each was injected per embryo. For all mRNA injections, a total of $1.6 \mathrm{ng}$ was injected per embryo. In coinjection experiments of XPtf1a/p48GR together with XlHbox8GR, $0.8 \mathrm{ng}$ of each was injected. For single injections, $0.8 \mathrm{ng}$ of the respective mRNA was injected together with $0.8 \mathrm{ng}$ of $\beta$-galactosidase RNA. Capped mRNAs were transcribed in vitro with the SP6 mMessage mMachine Kit (Ambion). All mRNAs and MOs were injected into the four vegetal cells of eight-cell-stage embryos from the vegetal pole. For the activation of the GR fusion proteins, dexamethasone (Sigma) was prepared as $5 \mathrm{mM}$ stock solution in $100 \%$ ethanol and applied to the control and mRNA-injected embryos at desired stages in a concentration of $10 \mu \mathrm{M}$ in $0.1 \times \mathrm{MBS}$. Embryos were kept in dexamethasone up to stage 41 .
Whole-mount in situ hybridization

Whole-mount in situ hybridization was done as described (Hollemann et al. 1999). The antisense probes for insulin, XPDIp, amylase, elastase, Cy118, XHex, XPtf1a/p48, and X1Hbox8 were prepared as described previously (Afelik et al. 2004; Chen et al. 2004). The antisense probe for Foxa1 was prepared from the full-length cDNA clone in pCS2+, which was cloned by RT-PCR based on the sequence from the data bank (GenBank accession nos. M93658 and BC047130).

\section{Immunohistochemistry}

Embryos were fixed in $4 \%$ formaldehyde for $1 \mathrm{~h}$. Immunohistochemistry staining was done on transverse paraffin sections $(5 \mu \mathrm{m})$ following the protocol described by Collombat et al. (2003). The following primary antibodies were used: mouse anti-glucagon, and guinea pig anti-insulin (1:1000; Sigma). The following secondary antibodies were used: Alexa Fluor488-conjugated anti-guineapig (1:1000; Invitrogen) and Cy3-conjugated anti-mouse (1:500; Dianova). The fluorescence images were documented with Axioplan 2 fluorescent microscope (Zeiss).

\section{Hematoxylin-eosin (HE) staining}

Paraffin sections $(5 \mu \mathrm{m})$ of embryos prefixed in Bouin's fixative were stained with hematoxylin and eosin (Sigma), following the protocol as described by Collombat et al. (2003).

\section{Vibratome sectioning}

Vibratome sections $(30 \mu \mathrm{m})$ were prepared as described previously using a Leica VT1000S Vibratome (Hollemann et al. 1998).

\section{Acknowledgments}

This work is supported by funds from the Deutsche Forschungsgemeinshaft (Pi159/8-1). S.A. is the recipient of a Georg Lichtenberg stipend. We are grateful to Marion Dornwell for technical assistance, Andreas Nolte for DNA sequencing, and Dr. Masashi Kawaichi for the mouse Ptf1a/p48 clone.

\section{References}

Afelik, S., Chen, Y., and Pieler, T. 2004. Pancreatic protein disulfide isomerase (XPDIp) is an early marker for the exocrine lineage of the developing pancreas in Xenopus laevis embryos. Gene Expr. Patterns 4: 71-76.

Ahlgren, U., Jonsson, J., and Edlund, H. 1996. The morphogenesis of the pancreatic mesenchyme is uncoupled from that of the pancreatic epithelium in IPF1/PDX1-deficient mice. Development 122: 14091416.

Ber, I., Shternhall, K., Perl, S., Ohanuna, Z., Goldberg, I., Barshack, I., Benvenisti-Zarum, L., Meivar-Levy, I., and Ferber, S. 2003. Functional, persistent, and extended liver to pancreas transdifferentiation. J. Biol. Chem. 278: 31950-31957.

Beres, T.M., Masui, T., Swift, G.H., Shi, L., Henke, R.M., and MacDonald, R.J. 2006. PTF1 is an organ-specific and Notch-independent basic helix-loop-helix complex containing the mammalian Suppressor of Hairless (RBP-J) or its paralogue, RBP-L. Mol. Cell. Biol. 26: 117-130.

Bort, R. and Zaret, K. 2002. Paths to the pancreas. Nat. Genet. 30: 85-86.

Chen, Y., Jürgens, K., Hollemann, T., Claußen, M., Ramadori, G., and Pieler, T. 2003. Cell-autonomous and signal-dependent expression of liver and intestine marker genes in endodermal precursor cells from Xenopus embryos. Mech. Dev. 120: 277-288.

Chen, Y., Pan, F.C., Brandes, N., Afelik, S., Sölter, M., and Pieler, T. 2004 Retinoic acid signalling is essential for pancreas development and promotes endocrine at the expense of exocrine cell differentiation in Xenopus. Dev. Biol. 271: 144-160.

Cockell, M., Stevenson, B.J., Strubin, M., Hagenbüchle, O., and Wellauer, P.K. 1989. Identification of a cell-specific DNA-binding activity that interacts with a transcriptional activator of genes expressed in the acinar pancreas. Mol. Cell. Biol. 9: 2464-2476.

Collombat, P., Mansouri, A., Hecksher-Sørensen, J., Serup, P., Krull, J., Gradwohl, G., and Gruss, P. 2003. Opposing actions of Arx and Pax4 in endocrine pancreas development. Genes \& Dev. 17: 2591-2603.

Ferber, S., Halkin, A., Cohen, H., Ber, I., Einav, Y., Goldberg, I., Barshack, I., Seijffers, R., Kopolovic, J., Kaiser, N., et al. 2000. Pancreatic and 


\section{Afelik et al.}

duodenal homeobox gene 1 induces expression of insulin genes in liver and ameliorates streptozotocin-induced hyperglycemia. Nat. Med. 6: $568-572$.

Gammill, L.S. and Sive, H. 1997. Identification of otx2 target genes and restrictions in ectodermal competence during Xenopus cement gland formation. Development 124: 471-481.

Grapin-Botton, A., Majithia, A.R., and Melton, D.A. 2001. Key events of pancreas formation are triggered in gut endoderm by ectopic expression of pancreatic regulatory genes. Genes \& Dev. 15: 444-454.

Gu, G., Dubauskaite, J., and Melton, D.A. 2002. Direct evidence for the pancreatic lineage: NGN3C cells are islet progenitors and are distinct from duct progenitors. Development 129: 2447-2457.

Hollemann, T., Chen, Y., Grunz, H., and Pieler, T. 1998. Regionalized metabolic activity establishes boundaries of retinoic acid signalling. EMBO J. 17: 7361-7372.

Hollemann, T., Panitz, F., and Pieler, T. 1999. In situ hybridization techniques with Xenopus embryos. In A comparative methods approach to the study of oocytes and embryos (ed. J.D. Richter), pp. 279-290. Oxford University Press, New York.

Horb, M.E. and Slack, J.M.W. 2002. Expression of amylase and other pancreatic genes in Xenopus. Mech. Dev. 113: 153-157.

Horb, M.E., Shen, C.N., Tosh, D., and Slack, J.M. 2003. Experimental conversion of liver to pancreas. Curr. Biol. 13: 105-115.

Jonsson, J., Carlsson, L., Edlund, T., and Edlund, H. 1994. Insulin-promoter-factor 1 is required for pancreas development in mice. Nature 371: 606-609.

Kawaguchi, Y., Cooper, B., Gannon, M., Ray, M., MacDonald, R.J., and Wright, C.V. 2002. The role of the transcriptional regulator Ptfla in converting intestinal to pancreatic progenitors. Nat. Genet. 32: 128134.

Kelly, O.G. and Melton, D.A. 2000. Development of the pancreas in Xenopus laevis. Dev. Dyn. 218: 615-627.

Kojima, H., Fujimiya, M., Matsumura, K., Younan, P., Imaeda, H., Maeda, M., and Chan, L. 2003. NeuroD-betacellulin gene therapy induces islet neogenesis in the liver and reverses diabetes in mice. Nat. Med. 9: 596-603.

Krapp, A., Knöfler, M., Ledermann, B., Burki, K., Berney, C., Zoerkler, N., Hagenbüchle, O., and Wellauer, P.K. 1998. The bHLH protein PTF1p48 is essential for the formation of the exocrine and the correct spatial organization of the endocrine pancreas. Genes \& Dev. 12: 3752-3763.

Lin, J.W., Biankin, A.V., Horb, M.E., Ghosh, B., Prasad, N.B., Yee, N.S., Pack, M.A., and Leach, S.D. 2004. Differential requirement for ptfla in endocrine and exocrine lineages of developing zebrafish pancreas. Dev. Biol. 274: 491-503.

Obata, J., Yano, M., Mimura, H., Goto, T., Nakayama, R., Mibu, Y., Oka, C., and Kawaichi, M. 2001. p48 subunit of mouse PTF1 binds to RBP-jk/CBF-1, the intracellular mediator of Notch signalling, and is expressed in the neural tube of early stage embryos. Genes Cells 6: 345-360.

Offield, M.F., Jetton, T.L., Labosky, P.A., Ray, M., Stein, R.W., Magnuson, M.A., Hogan, B.L., and Wright, C.V. 1996. PDX-1 is required for pancreatic outgrowth and differentiation of the rostral duodenum. Development 122: 983-995.

Ohlsson, H., Karlsson, K., and Edlund, T. 1993. IPF1, a homeodomaincontaining transactivator of the insulin gene. EMBO J. 12: 42514259.

Rupp, R., Snider, L., and Weintraub, H. 1994. Xenopus embryos regulate the nuclear localization of XMyoD. Genes \& Dev. 8: 1311-1323.

Slack, J.M. 1995. Developmental biology of the pancreas. Development 121: 1569-1580.

Yee, N.S., Yusuff, S., and Pack, M. 2001. Zebrafish pdx1 morphant displays defects in pancreas development and digestive organ chirality, and potentially identifies a multipotent pancreas progenitor cell. Genesis 30: 137-140.

Zecchin, E., Mavropoulos, A., Devos, N., Filippi, A., Tiso, N., Meyer, D., Peers, B., Bortolussi, M., and Argenton, F. 2004. Evolutionary conserved role of ptfla in the specification of exocrine pancreatic fates. Dev. Biol. 268: 174-184. 


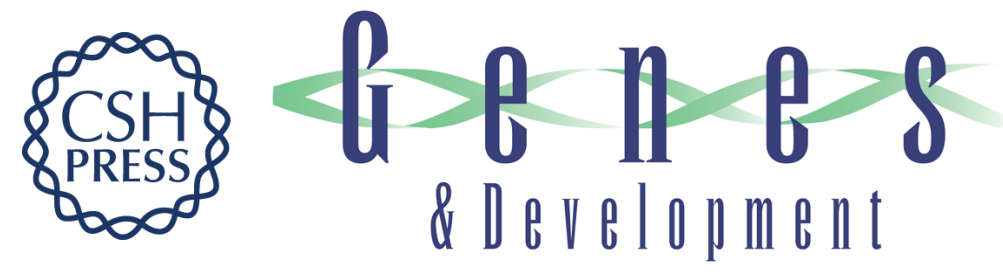

\section{Combined ectopic expression of Pdx1 and Ptf1a/p48 results in the stable conversion of posterior endoderm into endocrine and exocrine pancreatic tissue}

Solomon Afelik, Yonglong Chen and Tomas Pieler

Genes Dev. 2006, 20:

Access the most recent version at doi:10.1101/gad.378706

Supplemental http://genesdev.cshlp.org/content/suppl/2006/05/16/20.11.1441.DC1

Material

References This article cites 29 articles, 12 of which can be accessed free at:

http://genesdev.cshlp.org/content/20/11/1441.full.html\#ref-list-1

License

Email Alerting Receive free email alerts when new articles cite this article - sign up in the box at the top

Service right corner of the article or click here.

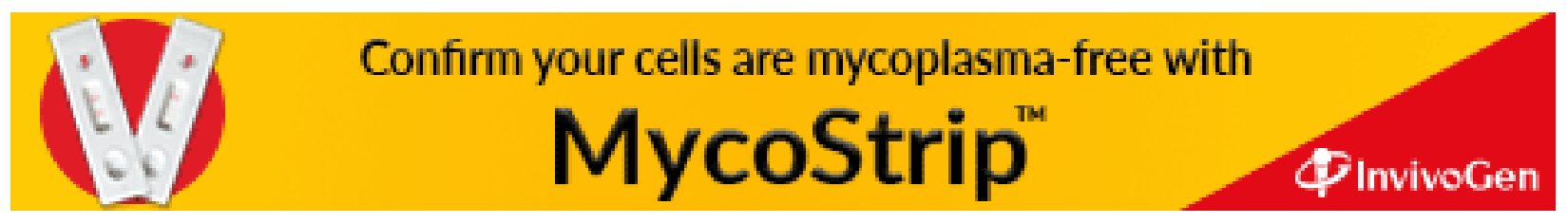

